GENE MAPPING

\section{Inequality of the sexes}

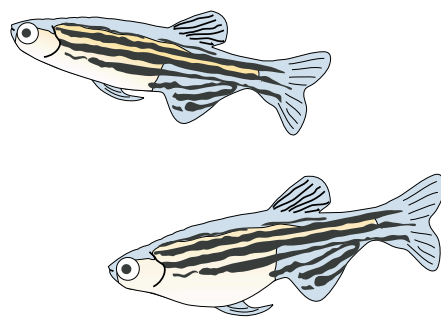

It's no secret that there are differences in how males and females behave, and it's no less of a secret among geneticists that these differences often extend all the way to their chromosomes. When it comes to the rate at which homologous chromosomes recombine during meiosis, for instance, one sex can outdo the other severalfold. The relative recombination rates in the two sexes aren't always easy to quantify genetic maps are often averaged across males and females - but such knowledge has useful implications for gene- mapping studies. This is why Singer and colleagues have embarked on a project to construct a meiotic map of the male zebrafish (Danio rerio), using the genetic trickery that only these fish can offer. The result - that male zebrafish recombine about three times less frequently than females — can be put to good use in designing experiments.

The map that the authors have constructed is based on the recombination frequency that occurs during male meiosis. This is the equivalent of looking at how well genetic markers have been reshuffled on single sperm derived from a parent that is heterozygous at many loci. Although such a direct experiment is beyond our current means, androgenetic haploid embryos, which contain only the paternal meiotic contribution, can be routinely generated in zebrafish. Singer et al. generated 94 such embryos from a father who was heterozygous for many polymorphic markers. The haploid embryos were genotyped at 185 of these markers, which were then placed on the male meiotic map. The result was unambiguous: across the 25 chromosomes, the male map is strikingly condensed (especially at the centromere), compared with a previously generated female meiotic map made with the same markers; the sex-average map lies almost exactly in-between.

So, what can we do now that we couldn't do before? As zebrafish continues to raise its profile as an experimental model for vertebrates, efficient gene mapping is becoming
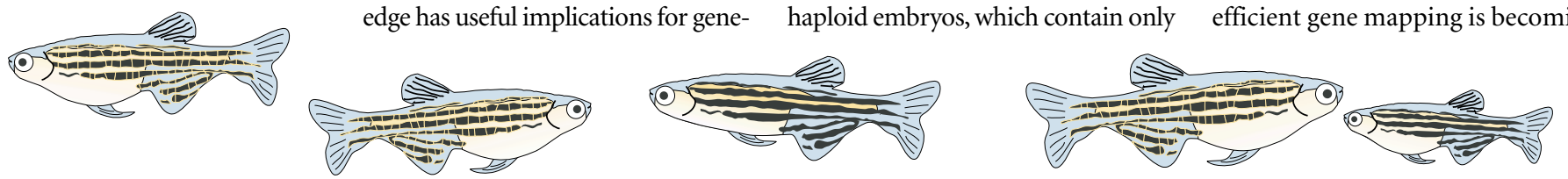

\section{dHand in hand with Gli3}

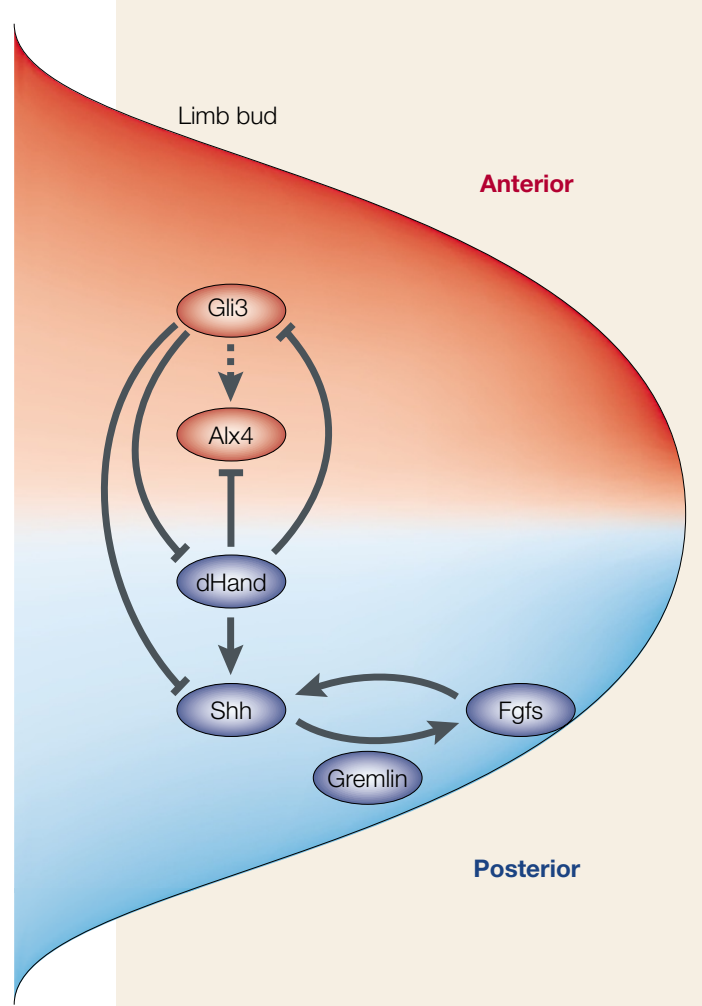

How vertebrate limbs are formed is considered one of the better understood processes in development. It is generally accepted that the early patterning of a vertebrate limb requires a feedback loop between sonic hedgehog ( $\mathrm{Shh}$ ) and fibroblast growth factor (Fgf) signals. But the limb bud is already patterned before this feedback loop is established, and the molecules responsible for this have until now remained unknown. New work by te Welscher and colleagues shows that the earlier patterning results from a mutual antagonism between two transcription factors - dHand and Gli3 — and that this interaction participates in positioning the Shh/Fgf signalling feedback loop.

During limb development, Shh signalling from the posterior mesenchyme regulates Fgf expression in the apical ectodermal ridge, whereas the Fgfs maintain Shh expression in the zone of polarizing activity. But Gremlin, a molecule that relays the Shh signal, and the Fgfs have asymmetric expression even before $S h h$ comes on, suggesting that the limb bud is prepatterned. To investigate this asymmetry, te Welscher and colleagues looked at a transcription factor, dHand, because it is required to establish Shh signalling and because its initially uniform expression becomes restricted to the posterior of the limb bud just before Shh activation - but how? The authors reasoned that answering this question would provide information about the earliest events in limb bud patterning. $S h h$ is repressed in the anterior of the limb bud by another transcription factor, Gli3. The authors found that, unlike in wild-type mice, dHand is expressed in the anterior mesenchyme of Gli3-deficient limbs, which suggests that dHand is repressed by Gli3. In the posterior limb bud mesenchyme, Gli3 is in turn repressed by dHand.

How do these early events relate to the Shh/Fgf-mediated patterning? As both Gremlin and the Fgfs are expressed before $S h h$, the authors looked at their expression in Gli3-deficient mice. It turns out that Gli3 is required to repress Gremlin and $\mathrm{Fgf4}$ in the anterior limb bud. The authors argue that the anterior expansion of Gremlin and Fgf4 in the absence of Gli3 could result from upregulating $d$ Hand in the anterior. Their hypothesis was confirmed by the anterior upregulation of Gremlin expression when $d H a n d$ was ectopically expressed.

Interestingly, the authors show that Alx4, 
an imperative. The high rates of recombination of females can be exploited for fine-mapping mutations. Conversely, if a more approximate map is desired, or if there is a need to preserve alleles in cis, then males, with their smaller map, would be preferred.

Why there is such discrepancy in recombination between the sexes we don't know, but this shouldn't stop us from using it to our advantage. Besides, where else can you get away with saying that males and females are suited for different tasks?

Tanita Casci

(2) References and links ORIGINAL RESEARCH PAPER Singer, A. et al. Sex-specific recombination rates in zebrafish. Genetics 160, 649-657 (2002)

John Postlethwait's lab:

http://www.neuro.uoregon.edu/postle/mydoc.htm

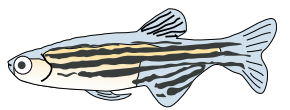

another transcription factor that is also expressed in anterior mesenchyme and participates in restricting Shh signalling, is not required for regulating $\mathrm{dHand}$ (or Gli3) expression. In fact, because $A l x 4$ expression is reduced in Gli3-deficient mice it must lie genetically downstream of Gli3.

This study provides important insight into the very early patterning events in the vertebrate limb, but before we have the complete picture of limb development, more factors will need to be incorporated into the already complex network of interactions. Magdalena Skipper

6) References and links ORIGINAL RESEARCH PAPER te Welscher, P. et al. Mutual genetic antagonism involving GLI3 and dHAND prepatterns the vertebrate limb bud mesenchyme prior to $\mathrm{SHH}$ signalling. Genes Dev. 16, 421-426 (2002) FURTHER READING Capdevila, J. \& Izpisua Belmonte, J. C. Patterning mechanisms controlling vertebrate limb mechanisms controlling vertebrate limb
development. Annu. Rev. Cell Dev. Biol. 17, development. Ann

WEB SITE

Encyclopedia of Life Sciences:

http://www.els.net

Vertebrate embryo: limb development
DEVELOPMENTAL BIOLOGY

\section{Sharpening the focus on Crumbs}

Thousands of people blinded by inherited retinal dystrophies harbour mutations in CRB1 which encodes a protein that is homologous to a regulator of Drosophila epithelial polarity called Crumbs. It has therefore been proposed that retinal epithelial cell disruption underlies the pathology of certain human retinal disorders such as retinitis pigmentosa and Leber congenital amaurosis - that are caused by $C R B 1$ mutations. Now, Pellikka $e t$ al. and Izaddoost $e t$ al. report in Nature that Crumbs has two specific roles in elaborating the architecture of photoreceptor cells (PRCs) that, surprisingly, occur well after early cell polarization. As these functions of Crumbs might be conserved in mammals, these findings shed new light on the cellular defects that underlie human blindness caused by $C R B 1$ mutations.

To overcome an early lethal phenotype and to maintain its function in the earlier stages of epithelial morphogenesis, both groups used mosaic analysis to remove Crumbs specifically in the developing retina of Drosophila. It was also overexpressed in fly PRCs. Previous studies had shown that Crumbs organizes the zonula adherens (ZA) — a band of 'glue' that encircles each epithelial cell and attaches the cells together in sheets. The studies reported here confirmed that Crumbs maintains ZA integrity in PRCs during the rapid expansion of the rhabdomere, an organelle that is packed with photosensitive membranes. During PRC differentiation, rhabdomeres (called the outer segment in vertebrates) elongate dramatically, and are supported by structures called stalks (or inner segments in vertebrates). Both groups noticed that loss of Crumbs in PRCs causes the abnormal positioning of the ZA and a striking shortening of rhabdomeres (see figure) but no significant loss of epithelial polarity. Izaddoost et al. showed that, when they overexpressed the intracellular domains of both Crumbs and CRB1 in Drosophila PRCs, the ZA was strikingly mislocalized. This suggested that Crumbs has a role in ZA formation that is distinct from its role in establishing epithelial polarity, which requires different structural domains of the protein and uses different protein partners.

When Pellikka et al. compared developing PRCs in fly and mouse retinas, they found that, once distinct rhabdomere (outer segment) and stalk (inner segment) membranes are established, Crumbs and CRB1 become
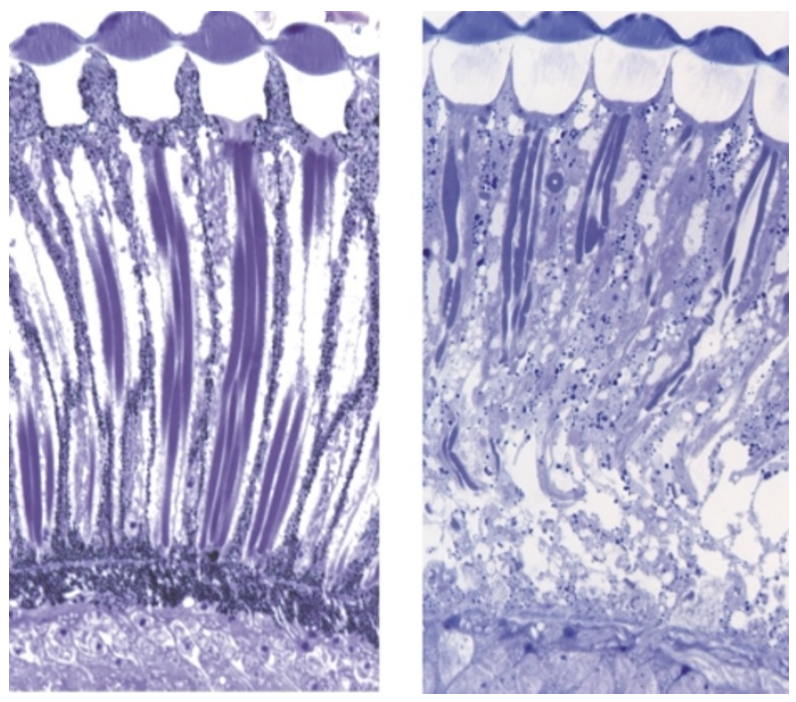

In wild-type adult fly retinas, rhabdomeres extend into long rod-like structures (left) but shorten by $50 \%$ of their normal length in the absence of Crumbs (right). Reproduced with permission from Pellikka, M. et al. (c) (2002) Macmillan Magazines Ltd.

restricted to the stalk and inner segment, respectively. Both groups report a role for Crumbs in rhabdomere elongation, and Pellikka et al. show that the extracellular domain of Crumbs has a role in stalk formation as well. First, they showed that loss of Crumbs shortened the stalks in flies, whereas overexpressing its extracellular domain during PRC development extended them. They also show that beta $[[\mathrm{H}]]$ spectrin physically associates with Crumbs and contributes to stalk elongation, possibly by negatively regulating endocytosis. Together, the studies suggest that, in developing PRCs, Crumbs binds through an intracellular domain to protein partners to position the ZA. Once the ZA is in place, it then interacts with different protein partners on the inner and outer surfaces of the cells to recruit membranes into the extending stalk.

Although the eyes of flies and humans are strikingly different in their morphologies and phototransduction cascades, these studies highlight similarities in photoreceptor morphogenesis and provide new models and approaches for uncovering the molecular interactions that might be involved in certain forms of human blindness.

Natalie DeWitt, Senior Editor, Nature

\section{8) References and links}

\section{ORIGINAL RESEARCH PAPERS}

Pellikka, M. et al. Crumbs, the Drosophila homologue of human CRB1/RP12, is essential for photoreceptor morphogenesis. Nature 17 February 2002 (DOI 10.1038/nature721) | Izaddoost, S. et a. Drosophila Crumbs is a positional cue in photoreceptor adherens junctions and rhabdomeres. Nature 17 February 2002 (DO) 10.1038/nature720)

\section{WEB SITES}

Kwang-Wook Choi's lab:

http://www bcm tmc edu/db/db fac-choi.html

Ulrich Tepass' lab: http://www.zoo.utoronto.ca/utepass/tepasshome 